\title{
Variación en los microartrópodos del suelo, por manejos forestales de raleo selectivo y cortes en hoyos de luz
}

\author{
Soil microarthropod variation as a result of selective thinning and round spot \\ clear-cutting forest management \\ RENE COVARRUBIAS ${ }^{1}$, ALVARO CONTRERAS $^{2}$ \\ ${ }^{1}$ Instituto de Entomología, Universidad Metropolitana de Ciencias de la Educación, \\ Casilla 147, Correo Central, Santiago, Chile. E-mail: nerrecovarru@yahoo.com \\ ${ }^{2}$ Instituto Forestal, Casilla 385, Valdivia, Chile.
}

\begin{abstract}
SUMMARY
A paper has already been written on the effects of forest management on the diversity and abundance of soil microarthropods in an evergreen forest of Chiloé (Chile). We detail a similar experiment, which shows the possible effects of two types of forest management (selective thinning and round spot clear-cutting) on the abundance and diversity of microarthropods in a different type of forest dominated by Nothofagus obliqua. This involved contrasting logged and old growth forests near Lanco, $10^{\text {th }}$ Region, Chile. Both impoverishment of microarthropod taxa and lower abundances of the dominant groups were recorded following the treatments.
\end{abstract}

Key words: microarthropods, Nothofagus forest, management, Chile.

\section{RESUMEN}

Ya existe un artículo acerca de los efectos de manejos forestales, sobre la diversidad y abundancia de microartrópodos del suelo, en un bosque chilote siempreverde en Chiloé, Chile. En el presente trabajo se muestran los resultados de una experiencia similar, en un tipo diferente de bosque, dominado por Nothofagus obliqua, en un sector cerca de Lanco, X Región (Chile), donde se contrastan partes inalteradas con otras en las que se realizaron dos tipos de tratamientos: raleo selectivo y cortes en hoyos o claros de luz. Se encontró disminución del número de taxa y baja en la abundancia de los grupos dominantes de microartrópodos en los tratamientos.

Palabras claves: microartrópodos, bosque Nothofagus, manejo, Chile.

\section{INTRODUCCION}

La utilización del bosque nativo sin acudir a su reemplazo total por especies maderables exóticas es en la actualidad un tema relevante. Los bosques templados húmedos del sur de Chile han sido alterados y reducidos en un $90 \%$ de su extensión original (1), siendo transformados en un mosaico de fragmentos boscosos, debido, entre otras causas, a los incendios, a la actividad forestal y dendroenergética. La fragmentación de los bosques nativos es una de las mayores amenazas contra la biodiversidad, lo que hace necesario desarrollar métodos silvoculturales que compatibilicen su conser- vación, con los beneficios económicos y sociales que esta actividad genera.

Para un manejo del bosque nativo son importantes, por una parte, su eventual rentabilidad y, por otra, los efectos ambientales provocados por las intervenciones silvícolas y cosecha. En Chile se han venido estudiando los efectos producidos por las intervenciones forestales en diversos tipos de bosque nativo, con sistemas tales como cortas de protección uniforme, cortas de protección en fajas y cortas en hoyos de luz $(2,3,4)$.

Teniendo en vista lo que debe ser una explotación sustentable del bosque nativo, la aplicación de estos sistemas no puede ser adoptada sin cono- 
cer los efectos que puedan provocar estos sistemas silviculturales. En relación al corte en franjas, Hawley \& Smith (5) estudiaron la distancia al borde protector y presentan las ventajas de este sistema para resolver diversos problemas silvícolas. Bajo el mismo sistema, Probst y Crows (6) han encontrado como efecto un aumento de la biodiversidad, por el hecho de combinar etapas sucesionales diferentes.

Respecto al método de protección en fajas Ocaña-Vidal (7) ha encontrado que presenta ventajas ecológicas y que genera condiciones adecuadas para la regeneración del bosque, en Perú y Costa Rica.

Entre los factores relevantes que se han estudiado en experiencias de silvicultura, está la temperatura del suelo (8), la temperatura del aire (9) y la luminosidad $(10,12)$.

La aplicación de diferentes sistemas de cosecha influye en la superficie del suelo debido a que algunos de ellos permiten una planificación de las operaciones más eficientes y además concentran la explotación, lo cual influirá también en el grado de compactación del suelo $(13,14)$; el caso de las huellas de madereo, como factor que incide en pérdidas de crecimiento de las plantas ha sido puesto en evidencia por Wert y Thomas (15) y Krag et al. (16). Los aumentos en la densidad del suelo han sido documentados por Gayoso e Iroumé (14) y por Malmer y Grip (17) en diversos tipos de suelo.

En Chile los renovales de bosque nativo constituyen un recurso de gran valor e importancia. La superficie de renovales en el país se estima en cerca de 2,9 millones de ha, entre renovales puros y mixtos. El origen de estos renovales está en explotaciones intensivas iniciales o como resultado de fenómenos naturales, tales como deslizamientos de suelo o avalanchas; según el origen, se forman renovales bajo dosel, o bien, formaciones a campo abierto. Sin embargo, el principal origen de estas formaciones estaría en los grandes incendios originados por el hombre hace 40 ó 50 años en el sur del país, como una forma de habilitar suelos para la actividad agropecuaria. Según INFOR (18), a principios de la década del 60, sólo en las provincias de Cautín y Valdivia existían 2,9 millones de hectáreas quemadas. Dentro de los renovales se destacan los bosques de Roble-RaulíCoihue, los renovales del tipo siempreverde de Canelo y el renoval mixto de especies como Ulmo, Tepa, Mañío y Arrayán.
Los estudios de crecimiento de estos renovales muestran una gran diversidad de situaciones; sin embargo, se ha establecido que los crecimientos naturales sin manejo, para especies como Roble, Raulí y Coihue, fluctúan entre 3 y $12,5 \mathrm{~m}^{3} / \mathrm{ha} / \mathrm{año}$ (19). Entre las ventajas de los manejos de raleo, se cuentan los menores costos de explotación y el mejor estado sanitario del recurso.

Entre los problemas que es necesario analizar, en cuanto a la explotación del bosque nativo, uno que ha tenido poca atención es el de la posible influencia que puedan tener los diversos manejos forestales sobre la diversidad de la fauna edáfica de microartrópodos, la que tiene, sin duda, importancia en la mantención de las propiedades del subsistema suelo.

Wallwork (20) cita una reducción en la diversidad de fauna del suelo, como efecto del corte de árboles maderables, producto de las diversas alteraciones, entre las que se cuentan una mayor llegada de luz en superficie, mayores fluctuaciones térmicas y cambios en la humedad del suelo, con problemas de desecación superficial, agregación en superficie de grandes cantidades de desechos orgánicos, como ramas, hojas, cortezas, compactación del suelo, por hombres y máquinas, etc. Huhta et al. (21) señalan que el efecto del corte de árboles (clear-cutting) en sectores de bosque es diferente, según el taxón de que se trate, encontrándose grupos más sensibles que otros a las alteraciones.

En Chile, los efectos de manejos en general sobre los microartrópodos han sido estudiados sólo en algunos trabajos forestales $(2,3,4)$. Covarrubias (22) entrega datos sobre el efecto del reemplazo de bosque nativo por plantaciones de Pinus radiata, en donde, junto a diversos tipos de alteración, se incluye una disminución de la diversidad de taxa supraespecíficos de microartrópodos. Covarrubias y Contreras (23) encuentran diversas alteraciones en las poblaciones de microartrópodos, que se pueden atribuir a los tratamientos forestales de cortes en fajas y cortes en hoyos de luz, en bosque de Chiloé siempreverde, dando cuenta de alteraciones en la biodiversidad y abundancia, en detalle para todos los taxa supraespecíficos encontrados.

\section{MATERIAL Y METODOS}

En el presente trabajo se detalla un estudio de los microartrópodos del suelo, en áreas que han 
BOSQUE 25(1): 103-116, 2004

Variación en los microartrópodos del suelo, por manejos forestales de raleo selectivo y cortes en hoyos de luz

sido sometidas a dos tipos de manejos forestales, contrastándolos con un sector inalterado, como testigo o control; el sector del estudio se denomina Lilcoco (72³9'30" Long. W y 39²8'24" Lat. S), distante a 10 kilómetros al este del pueblo de Lanco, X Región, Chile.

El clima corresponde a templado lluvioso, caracterizado por una alta precipitación y por un descenso de la temperatura hacia el sur. La precipitación media anual registrada en la estación más cercana, ubicada en Loncoche, es de $2.402 \mathrm{~mm}$ y la temperatura media anual es de $14,2^{\circ} \mathrm{C}$.

Los suelos del área pertenecen a la serie Lastarria (LAS); son suelos que corresponden a trumaos de cerro y se presentan en el cordón del complejo metamórfico de la costa, que se extiende en dirección oeste-este, al norte del pueblo de Lanco.

La vegetación existente corresponde a un bosque mixto de segundo crecimiento, caracterizado por la dominancia de Nothofagus obliqua (Mirb.), acompañados por Lomatia hirsuta (LAM.), Eucryphia cordifolia Cav., Gevuina avellana Mol., Laurelia sempervirens (R. et Pav.), Persea lingue Nees., Aextoxicon punctatum (R. et Pav.) y Caldcluvia paniculata (Cav.). El bosque presenta un área basal de 48,36 m²/ha, con 2.033 árboles/ha; la altura media es de $24 \mathrm{~m}$ y el diámetro medio cuadrático de $17,4 \mathrm{~cm}$; la edad aproximada del rodal es de 45 años.

Los sistemas de intervención que se estudiaron son los siguientes:

1. Las cortas de raleo de "árbol futuro" se realizan mediante la selección para la cosecha final de aproximadamente 300 árboles por hectárea, distribuidos homogéneamente; estos árboles son liberados de la competencia por copas, para lo cual se extraen árboles industrializables que compitan con él, quedando en pie también árboles acompañantes que ayudan al desarrollo de los futuros y son fuente de materia prima en próximos raleos.

En el presente caso se habían cortado todos los arbustos y uno de cada cuatro árboles, dejando en pie aquellos de mejor crecimiento y hábito.

2. Las cortas de protección en hoyos de luz o protección en grupos constituyen una variante del método de raleo, donde el efecto del dosel lateral permite regular las condiciones ambientales al interior del área intervenida. Se trata de un sistema que imita los procesos naturales, que forman claros de bosque, tales como distribuciones provocadas por la caída de grandes árboles por el viento, permitiendo con ello el desarrollo de regeneración. En este sistema de cortas se despejó a tala rasa una superficie circular, cuyo diámetro equivale a una vez la altura del dosel superior ( $25 \mathrm{~m}$ ), quedando como protección lateral bosque sin intervenir, de un ancho igual a la altura del dosel superior.

Los tratamientos se habían hecho en enero 1996 y en el caso de los hoyos de luz, después de hechos, fueron replantados.

Muestreo de microartrópodos: los muestreos para fauna del suelo se realizaron el 28 de agosto de 1997. En área de bosque testigo y de tratamiento de raleo, se tomaron 20 muestras aleatorias de suelo en cada uno; en el tratamiento hoyos de luz, se tomaron 10 muestras en un hoyo de luz (= hoyo-1), replantado con robles y raulíes, que en la fecha de muestreo tenían aproximadamente $1 \mathrm{~m}$ de alto y otras 10 muestras en otro hoyo (= hoyo-2), cercano y semejante, pero replantado sólo en fecha más reciente con robles, que el día del muestreo alcanzaban $50 \mathrm{~cm}$ de altura, siendo poco visibles entre las ramas caídas y desechos de la tala inicial remanentes en gran cantidad. Cada muestra consistió en hojarasca y humus, que se tomaron con pala de mano, entre 0 y $10 \mathrm{~cm}$ de profundidad, puestas en bolsas de polietileno e individualizadas. En el laboratorio se midieron volúmenes de 100 ml de cada una de las 60 muestras, los que se pusieron en los respectivos embudos de BerleseTullgren durante una semana, con iluminación por ampolletas de $40 \mathrm{~W}$; los microartrópodos se recogieron en tubos con alcohol $75^{\circ}$. Con el examen cuidadoso y exhaustivo del contenido de cada tubo se hizo un protocolo, consignando los grupos zoológicos presentes a nivel de taxa supraespecíficos y el número de individuos encontrados en cada grupo. Todo el examen de microartrópodos se realizó bajo microscopio binocular estereoscópico. En todas las muestras de suelo también se midió el contenido de agua de las muestras, por diferencia de peso antes y después de una desecación a $105^{\circ} \mathrm{C}$ durante dos horas.

En los métodos estadísticos se empleó análisis de varianza a un criterio de clasificación, con transformación de los datos a $y=\log x+1$, para compensar la heterogeneidad de las varianzas (24). 
BOSQUE 25(1): 103-116, 2004

Variación en los microartrópodos del suelo, por manejos forestales de raleo selectivo y cortes en hoyos de luz

Las mediciones de diversidad (índice H') y equiparidad (índice J) se describen en Pielou (25).

\section{RESULTADOS Y DISCUSION}

Contenido de agua y peso seco en las muestras: peso seco y contenido de agua, en las muestras de bosque testigo; manejos de raleo selectivo y manejo de corte circular en hoyos de luz; se aprecian en el cuadro 1; también se muestran los promedios de agua expresados como porcentaje del peso seco.

Llama la atención la elevada cantidad de agua, del orden de 130 a $135 \%$ de peso seco que la puede retener; sin embargo, esto no es raro, al comparar con los contenidos de agua en otros tipos de bosque; por ejemplo, en bosques de Lenga (Nothofagus pumilio) (26) o en bosques de ñadi, tipo renoval de canelo, en Chiloé (23), donde se encuentran cifras aún mayores.

Se probó mediante análisis de varianza si había diferencias entre pesos secos, o en contenidos de agua, entre bosque testigo y tratamientos, así como entre dos subgrupos de hoyos diferentes y cercanos, los resultados se pueden ver en el cuadro 2 .

Se puede observar que no hay diferencias significativas para pesos secos, en ningún contraste del tipo testigo/tratamiento, incluyendo análisis separados para los dos subgrupos de hoyos, ni tampoco entre estos dos últimos. Estos datos sugieren una homogeneidad de los componentes secos del suelo en todo el sitio de mediciones.
El contenido de agua sí difiere significativamente entre el bosque testigo y el manejo de cortes circulares (hoyos), tendencia que se mantiene al contrastar el grupo testigo individualmente con los subgrupos hoyo-1 y hoyo- 2 .

El contraste entre los dos subgrupos hoyo-1/ hoyo-2 no es significativo, por lo que, en este aspecto, se pueden considerar como una sola unidad.

El sentido de las diferencias encontradas se traduce en que la media de contenido de agua es mayor en muestras de hoyos $(31,08 \mathrm{~g})$ que en las de bosque testigo $(27,16)$. La causa de este fenómeno podría ser una mayor caída directa de lluvia al suelo, por falta de árboles que produzcan intercepción, lo que no es el caso en bosque testigo; apoyando esta hipótesis está el dato de que este fenómeno no se repite en el tratamiento "raleo" que, al no eliminar el total de árboles, permite aun la intercepción de las copas y ramas en un porcentaje apreciable; de hecho los contenidos de agua en suelos de bosque testigo y de tratamiento raleo no son significativamente diferentes.

En el caso de mayor cantidad de agua en "hoyos", ésta podría deberse también a mayor condensación de neblina que entraría en contacto directamente con el suelo, o también a la presencia momentánea de una mayor cantidad de materia orgánica suelta, fruto de las labores de corte de árboles iniciales del tratamiento (ramas, hojas, corteza, aserrín).

\section{CUADRO 1}

Peso seco y contenido de agua en muestras de suelo de bosque de Nothofagus obliqua, testigo y tratamientos. Medias, desviaciones estándar y porcentaje de agua sobre el peso seco.

Dry weight and water content in soil samples from a Nothofagus obliqua forest. Treated and control plots. Arithmetic means, standard deviations, \% water/dry weight.

\begin{tabular}{|l|c|c|c|c|c|}
\cline { 2 - 5 } \multicolumn{1}{c|}{} & \multicolumn{2}{c|}{$\begin{array}{c}\text { Peso seco } \\
\text { (gramos) }\end{array}$} & \multicolumn{2}{c|}{$\begin{array}{c}\text { Contenido agua } \\
\text { (gramos) }\end{array}$} & $\begin{array}{c}\% \\
\text { promedio agua, } \\
\text { sobre peso seco }\end{array}$ \\
\cline { 2 - 5 } & Media & Desv. estándar & Media & Desv. estándar & \\
\hline Testigo: bosque nativo & 20,89 & 5,61 & 27,16 & 2,67 & 130,01 \\
Tratamiento raleo & 20,30 & 6,65 & 27,30 & 3,28 & 134,48 \\
Tratamiento hoyo & 23,01 & 6,06 & 31,08 & 3,38 & 135,07 \\
\hline
\end{tabular}


BOSQUE 25(1): 103-116, 2004

Variación en los microartrópodos del suelo, por manejos forestales de raleo selectivo y cortes en hoyos de luz

\section{CUADRO 2}

Resultado de análisis de varianza, para valores de pesos secos y de contenidos de agua en suelos de bosque testigo y de dos tratamientos.

Results of the analysis of variance, using dry weights and water content as variables, in treated (selective thinning and round spot clear-cutting) and control plots.

\begin{tabular}{|l|c|c|c|c|c|c|}
\cline { 2 - 7 } & \multicolumn{3}{|c|}{ Peso seco } & \multicolumn{3}{c|}{ Contenido de Agua } \\
\cline { 2 - 7 } & F & $\begin{array}{c}\text { Grados } \\
\text { libertad }\end{array}$ & $\begin{array}{c}\text { Signifi- } \\
\text { cación }\end{array}$ & F & $\begin{array}{c}\text { Grados } \\
\text { libertad }\end{array}$ & $\begin{array}{c}\text { Signifi- } \\
\text { cación }\end{array}$ \\
\hline Testigo/Hoyos & 0,06 & 1 y 38 & NS & 16,45 & 1 y 38 & SSS \\
Testigo/Raleo & 0,09 & 1 y 38 & NS & 0,02 & 1 y 38 & NS \\
Testigo/Hoyo-1 & 0,86 & 1 y 28 & NS & 14,47 & 1 y 18 & SS \\
Testigo/Hoyo-2 & 0,56 & 1 y 28 & NS & 4,46 & 1 y 18 & S \\
Hoyo1/Hoyo-2 & 0,05 & 1 y 18 & NS & 1,03 & 1 y 18 & NS \\
\hline
\end{tabular}

$\mathrm{SSS}=\mathrm{p}<0,001 ; \mathrm{SS}=\mathrm{p}<0,01 ; \mathrm{S}=\mathrm{p}<0,05 ; \mathrm{NS}=\mathrm{P}>0,05$.

Taxa encontrados, abundancia y frecuencia: para bosque testigo y para cada sector tratamiento, los taxa de microartrópodos obtenidos de los grupos muestrales, su frecuencia de presentación en las muestras, expresada en porcentaje; sus densidades medias, expresadas como número de individuos por $1.000 \mathrm{ml}$ de material y las respectivas desviaciones estándar (cuadros 3, 4, 5, 6 y 7); también se muestra el número absoluto de individuos colectados y clasificados, base del estudio. En el caso de Hoyos se dan los datos para el conjunto (cuadro 5) como por separado (cuadros 6 y 7).

Para el análisis elegimos un primer grupo de taxa por tener la propiedad de presentar frecuencias muestrales elevadas (> $80 \%$ ), lo que permite un estudio cuantitativo, que se tratará en un capítulo aparte. En bosque testigo y tratamiento raleo estos grupos son: ácaros Oribatida y Gamasina, total de Acarina, Collembola Entomobryomorpha y Poduromorpha, así como el total de fauna. Como ocurre en la gran mayoría de los casos estos taxa frecuentes son los que también tienen densidades medias más altas.

Un segundo grupo de taxa incluye aquellos con frecuencias inferiores al primero, que en general se mostraron no mayores a 50\%, quedando aquí ácaros Prostigmata y Uropodina, Pseudoscorpionida, larvas de Díptera y de Coleóptera.

Un tercer grupo incluye una miscelánea de taxa de abundancias muy bajas, ninguno de los cuales presentó más de 15 individuos en el total de las muestras, y en 13 de los 15 casos se encontraron en total sólo 1 ó 2 individuos.

En cuanto a los dos grupos de bajas frecuencias y abundancias, sirven de apoyo para señalar las siguientes diferencias cualitativas entre testigo y tratamientos.

Hay un grupo de taxa que están presentes en bosque testigo y ausentes en todos los tratamientos: Thysanóptera, Coleóptera, Dermestidae, Tenebrionidae y Carabidae; además, otros están ausentes en tratamiento hoyo: larvas de Lepidóptera, Copépoda e Isópoda y finalmente otros están ausentes sólo en tratamiento raleo: Campodeidae, Hemíptera y Coleóptera Leiodidae. No se comentó sobre la presencia ocasional de pupas de Formicidae o imagos de Díptera, porque el método de Berlese no permite hacer observaciones representativas sobre ellos.

Otra observación es que en 13 taxa se presentan abundancias mayores en bosque testigo (Tarsonemini, Uropodina, Pseudoscorpionida, Chilópoda, Thysanóptera, Coleóptera Leiodidae y Dermestidae, larvas de Díptera, de Coleóptera y de Lepidóptera, Campodeidae y los crustáceos Isópoda y Copépoda), la presencia de Copépoda constituye prueba de las condiciones de elevada humedad en el suelo; sin embargo, no se encontraron en tratamiento hoyo. 
BOSQUE 25(1): 103-116, 2004

Variación en los microartrópodos del suelo, por manejos forestales de raleo selectivo y cortes en hoyos de luz

\section{CUADRO 3}

Microartrópodos del suelo en bosque testigo. Frecuencia de presentación en muestras (\%), densidad media (en $\mathrm{N}^{\circ}$ individuos $/ 1.000 \mathrm{ml}$ ), desviación estándar y número total de individuos colectados.

Soil microarthropods in control forest plots. Sample frequencies (5), mean densities $\left(\mathrm{N}^{\mathrm{r}}\right.$ individuals $\left./ 1,000 \mathrm{ml}\right)$, standard deviations and total numbers of collected individuals.

\begin{tabular}{|c|c|c|c|c|}
\hline $\begin{array}{l}\text { Lanco-C } \\
\text { Bosque testigo }\end{array}$ & $\begin{array}{c}\text { Frecuencia } \\
\%\end{array}$ & $\begin{array}{c}\mathrm{N}^{\mathrm{o}} \\
\text { individuos/ } \\
1.000 \mathrm{ml} \\
\text { Medias }\end{array}$ & $\begin{array}{l}\text { Desviación } \\
\text { estándar }\end{array}$ & $\begin{array}{c}\mathrm{N}^{\mathrm{o}} \\
\text { total } \\
\text { individuos } \\
\text { colectados }\end{array}$ \\
\hline Oribatida & 100 & 377,0 & 218,9 & 754 \\
\hline Prostigmata & 55 & 8,0 & 10,1 & 16 \\
\hline Tarsonemini & 35 & 8,5 & 16,9 & 17 \\
\hline Gamasina & 100 & 78,5 & 54,0 & 15,7 \\
\hline Uropodina & 70 & 46,0 & 74,4 & 92 \\
\hline Total Acarina & 100 & 518,0 & 277,0 & 1.036 \\
\hline Araneida & 15 & 1,5 & 3,7 & 3 \\
\hline Pseudoscorpionida & 50 & 8,5 & 11,4 & 17 \\
\hline Diplópoda & 20 & 2,0 & 4,1 & 4 \\
\hline Chilópoda & 25 & 4,5 & 8,9 & 9 \\
\hline Collembola Entomobryomorpha & 100 & 79,0 & 91,4 & 158 \\
\hline Collembola Podurmorpha & 85 & 41,5 & 41,3 & 8,3 \\
\hline Collembola Symphypleona & 15 & 3,5 & 9,9 & 7 \\
\hline Hemíptera & 5 & 1,0 & 4,5 & 2 \\
\hline Thysanóptera & 10 & 1,5 & 4,9 & 3 \\
\hline Coleóptera Staphylinidae & 35 & 4,0 & 6,0 & 8 \\
\hline Coleóptera Leiodidae & 20 & 2,5 & 5,5 & 5 \\
\hline Coleóptera Ptiliidae & & & & \\
\hline Coleóptera Curculionidae & 15 & 1,5 & 3,7 & 3 \\
\hline Coleóptera Pselaphidae & 5 & 0,5 & 2,2 & 1 \\
\hline Coleóptera Tenebrionidae & 5 & 0,5 & 2,2 & 1 \\
\hline Coleóptera Dermestidae & 5 & 0,5 & 2,2 & 1 \\
\hline Coleóptera Carabidae & 5 & 0,5 & 2,2 & 1 \\
\hline Larvas Díptera & 75 & 18,5 & 20,6 & 37 \\
\hline Larvas Coleóptera & 70 & 12,5 & 10,7 & 25 \\
\hline Larvas Lepidóptera & 25 & 2,5 & 4,4 & 5 \\
\hline Isópoda & 35 & 5,5 & 11,5 & 11 \\
\hline Copépoda & 35 & 5,0 & 8,3 & 10 \\
\hline Díptera (Imago) & - & - & - & - \\
\hline Formicidae & 5 & 0,5 & 2,2 & 1 \\
\hline Diplura Campodeidae & 20 & 3,5 & 8,1 & 7 \\
\hline Total Fauna & 100 & 719,0 & 372,3 & 1.430 \\
\hline $\mathrm{N}^{\circ}$ taxa: 28 & & & & \\
\hline
\end{tabular}


BOSQUE 25(1): 103-116, 2004 Variación en los microartrópodos del suelo, por manejos forestales de raleo selectivo y cortes en hoyos de luz

\section{CUADRO 4}

Microartrópodos del suelo en tratamiento "Raleo de árbol futuro". Frecuencia de presentación en muestras (\%).densidad media en $\mathrm{N}^{\circ}$ individuos $/ 1.000 \mathrm{ml}$, desviación estándar y número total de individuos colectados. Soil microarthropods in selectively thinned treated forest plots. Sample frequencies (\%), mean densities $\left(\mathrm{N}^{\mathrm{r}}\right.$ individuals $\left./ 1,000 \mathrm{ml}\right)$, standard deviations and total number of collected individuals.

\begin{tabular}{|c|c|c|c|c|}
\hline $\begin{array}{l}\text { Lanco-C } \\
\text { Raleo }\end{array}$ & $\begin{array}{c}\text { Frecuencia } \\
\%\end{array}$ & $\begin{array}{c}\mathrm{N}^{\mathrm{o}} \\
\text { individuos/ } \\
1.000 \mathrm{ml} \\
\text { Medias }\end{array}$ & $\begin{array}{c}\text { Desviación } \\
\text { estándar }\end{array}$ & $\begin{array}{c}\mathrm{N}^{\mathrm{o}} \\
\text { total } \\
\text { individuos } \\
\text { colectados }\end{array}$ \\
\hline Oribatida & 100 & 168,5 & 121,5 & 337 \\
\hline Prostigmata & 30 & 4,5 & 7,6 & 9 \\
\hline Tarsonemini & 15 & 3,0 & 8,0 & 6 \\
\hline Gamasina & 85 & 47,5 & 44,8 & 95 \\
\hline Uropodina & 45 & 19,5 & 25,2 & 39 \\
\hline Total Acarina & 100 & 243,0 & 153,5 & 486 \\
\hline Araneida & 5 & 0,5 & 2,2 & 1 \\
\hline Pseudoscorpionida & 45 & 7,5 & 10,2 & 15 \\
\hline Diplópoda & 5 & 0,5 & 2,2 & 1 \\
\hline Chilópoda & 5 & 0,5 & 2,2 & 1 \\
\hline Collembola Entomobryomorpha & 80 & 46,0 & 54,9 & 92 \\
\hline Collembola Podurmorpha & 60 & 23,0 & 38,7 & 46 \\
\hline Collembola Symphypleona & 35 & 7,5 & 11,2 & 15 \\
\hline Hemíptera & - & - & - & - \\
\hline Thysanóptera & - & - & - & - \\
\hline Coleóptera Staphylinidae & 45 & 6,0 & 8,2 & 12 \\
\hline Coleóptera Leiodidae & - & - & - & - \\
\hline Coleóptera Ptiliidae & 30 & 3,0 & 4,7 & 6 \\
\hline Coleóptera Curculionidae & 10 & 1,0 & 3,1 & 2 \\
\hline Coleóptera Pselaphidae & 5 & 0,5 & 2,2 & 1 \\
\hline Coleóptera Tenebrionidae & - & - & - & - \\
\hline Coleóptera Dermestidae & - & - & - & - \\
\hline Coleóptera Carabidae & - & - & - & - \\
\hline Larvas Díptera & 55 & 8,5 & 9,3 & 17 \\
\hline Larvas Coleóptera & 55 & 10,5 & 12,3 & 21 \\
\hline Larvas Lepidóptera & 5 & 0,5 & 2,2 & 1 \\
\hline Isópoda & 10 & 2,5 & 7,9 & 5 \\
\hline Copépoda & 5 & 0,5 & 2,2 & 1 \\
\hline Díptera (Imago) & - & - & - & - \\
\hline Formicidae & - & - & - & - \\
\hline Diplura Campodeidae & - & - & - & - \\
\hline Total Fauna & 100 & 3.615 & 217,0 & 723 \\
\hline $\mathrm{N}^{\mathrm{o}}$ taxa: 21 & & & & \\
\hline
\end{tabular}


BOSQUE 25(1): 103-116, 2004

Variación en los microartrópodos del suelo, por manejos forestales de raleo selectivo y cortes en hoyos de luz

\section{CUADRO 5}

Microartrópodos del suelo en tratamiento "hoyo de luz". Frecuencia de presentación en muestras (\%), densidad media en $\mathrm{N}^{\mathrm{o}}$ individuos/1.000 ml, desviación estándar (s) y número total de individuos colectados.

Soil microarthropods in round spot clear-cut treated forest plots. Sample frequencies $(\%)$, mean densities $\left(\mathrm{N}^{\mathrm{r}}\right.$ individuals $\left./ 1,000 \mathrm{ml}\right)$, standard deviations and total number of collected individuals.

\begin{tabular}{|c|c|c|c|c|}
\hline $\begin{array}{l}\text { Lanco-H } \\
\text { Hoyo } 1+\text { Hoyo } 2\end{array}$ & $\begin{array}{c}\text { Frecuencia } \\
\%\end{array}$ & $\begin{array}{c}\mathrm{N}^{\circ} \\
\text { individuos/ } \\
1.000 \mathrm{ml} \\
\text { Medias }\end{array}$ & $\begin{array}{l}\text { Desviación } \\
\text { estándar }\end{array}$ & $\begin{array}{c}\mathrm{N}^{\mathrm{o}} \\
\text { total } \\
\text { individuos } \\
\text { colectados }\end{array}$ \\
\hline Oribatida & 100 & 216,5 & 154,52 & 433 \\
\hline Prostigmata & 40 & 5,5 & 7,59 & 11 \\
\hline Tarsonemini & 30 & 7,0 & 14,18 & 14 \\
\hline Gamasina & 95 & 49,5 & 45,36 & 99 \\
\hline Uropodina & 25 & 5,0 & 10,0 & 10 \\
\hline Total Acarina & 100 & 283,5 & 179,1 & 567 \\
\hline Araneida & 5 & 0,5 & 2,24 & 1 \\
\hline Pseudoscorpionida & 10 & 1,0 & 3,08 & 2 \\
\hline Diplópoda & 20 & 3,0 & 7,33 & 6 \\
\hline Chilópoda & 20 & 2,5 & 5,5 & 5 \\
\hline Collembola Entomobryomorpha & 75 & 42,5 & 64,1 & 85 \\
\hline Collembola Podurmorpha & 90 & 106,0 & 134,5 & 212 \\
\hline Collembola Symphypleona & 35 & 4,5 & 6,9 & 9 \\
\hline Hemíptera & 25 & 4,5 & 8,9 & 9 \\
\hline Thysanóptera & - & - & - & - \\
\hline Coleóptera Staphylinidae & 15 & 2,5 & 7,2 & 5 \\
\hline Coleóptera Leiodidae & 15 & 1,5 & 3,7 & 3 \\
\hline Coleóptera Ptiliidae & - & - & - & - \\
\hline Coleóptera Curculionidae & 5 & 0,5 & 2,24 & 1 \\
\hline Coleóptera Pselaphidae & 5 & 0,5 & 2,24 & 1 \\
\hline Coleóptera Tenebrionidae & - & - & - & - \\
\hline Coleóptera Dermestidae & - & - & - & - \\
\hline Coleóptera Carabidae & - & - & - & - \\
\hline Larvas Díptera & 80 & 27,0 & 27,0 & 54 \\
\hline Larvas Coleóptera & 45 & 8,0 & 11,1 & 16 \\
\hline Larvas Lepidóptera & - & - & - & - \\
\hline Isópoda & - & - & - & - \\
\hline Copépoda & - & - & - & - \\
\hline Díptera (Imago) & 5 & 0,5 & 2,2 & 1 \\
\hline Formicidae & 30 & 3,0 & 4,7 & 6 \\
\hline Diplura Campodeidae & 5 & 0,5 & 2,2 & 1 \\
\hline Total Fauna & 100 & 492 & 321,8 & 984 \\
\hline $\mathrm{N}^{\circ}$ taxa: 22 & & & & \\
\hline
\end{tabular}


BOSQUE 25(1): 103-116, 2004 Variación en los microartrópodos del suelo, por manejos forestales de raleo selectivo y cortes en hoyos de luz

\section{CUADRO 6}

Microartrópodos del suelo en tratamiento "hoyo 1". Frecuencia de presentación en muestras (\%), densidad media en $\mathrm{N}^{\circ}$ individuos $/ 1.000 \mathrm{ml}$, desviación estándar y número total de individuos colectados. Soil microarthropods in round spot clear-cut treated forest plots. Sample frequencies (\%), mean densities $\left(\mathrm{N}^{\mathrm{r}}\right.$ individuals $\left./ 1,000 \mathrm{ml}\right)$, standard deviations and total number of collected individuals.

\begin{tabular}{|c|c|c|c|c|}
\hline $\begin{array}{l}\text { Lanco-H } \\
\text { Ноyo } 1\end{array}$ & $\begin{array}{c}\text { Frecuencia } \\
\%\end{array}$ & $\begin{array}{c}\mathrm{N}^{\circ} \\
\text { individuos/ } \\
1.000 \mathrm{ml} \\
\text { Medias }\end{array}$ & $\begin{array}{l}\text { Desviación } \\
\text { estándar }\end{array}$ & $\begin{array}{c}\mathrm{N}^{\mathrm{o}} \\
\text { total } \\
\text { individuos } \\
\text { colectados }\end{array}$ \\
\hline Oribatida & 100 & 286,0 & 178,6 & 286 \\
\hline Prostigmata & 30 & 5,0 & 8,5 & 5 \\
\hline Tarsonemini & 50 & 13,0 & 18,3 & 13 \\
\hline Gamasina & 90 & 67,0 & 51,4 & 67 \\
\hline Uropodina & 10 & 3,0 & 9,49 & 3 \\
\hline Total Acarina & 100 & 374,0 & 196,0 & 374 \\
\hline Araneida & - & - & - & - \\
\hline Pseudoscorpionida & - & - & - & - \\
\hline Diplópoda & 10 & 3,0 & 9,5 & 3 \\
\hline Chilópoda & 30 & 4,0 & 7,0 & 4 \\
\hline Collembola Entomobryomorpha & 70 & 44,0 & 74,0 & 44 \\
\hline Collembola Podurmorpha & 10 & 172,0 & 159,6 & 172 \\
\hline Collembola Symphypleona & 60 & 8,0 & 7,9 & 8 \\
\hline Hemíptera & - & - & - & - \\
\hline Thrysanóptera & - & - & - & - \\
\hline Coleóptera Staphylinidae & 10 & 3,0 & 9,5 & 3 \\
\hline Coleóptera Leiodidae & - & - & - & - \\
\hline Coleóptera Ptiliidae & - & - & - & - \\
\hline Coleóptera Curculionidae & - & - & - & - \\
\hline Coleóptera Pselaphidae & 10 & 1,0 & 3,2 & 1 \\
\hline Coleóptera Tenebrionidae & - & - & - & - \\
\hline Coleóptera Dermestidae & - & - & - & - \\
\hline Coleóptera Carabidae & - & - & - & - \\
\hline Larvas Díptera & 80 & 33,0 & 30,6 & 33 \\
\hline Larvas Coleóptera & 40 & 9,0 & 13,7 & 9 \\
\hline Larvas Lepidóptera & - & - & - & - \\
\hline Isópoda & 10 & 1,0 & 3,2 & 1 \\
\hline Copépoda & - & - & - & - \\
\hline Díptera (Imago) & 80 & 33,0 & 30,6 & 33 \\
\hline Formicidae & 10 & 1,0 & 3,2 & 1 \\
\hline Diplura Campodeidae & - & - & - & - \\
\hline Total Fauna & 100 & 653,0 & 350,2 & 653 \\
\hline $\mathrm{N}^{\mathrm{o}}$ taxa: 15 & & & & \\
\hline
\end{tabular}


BOSQUE 25(1): 103-116, 2004

Variación en los microartrópodos del suelo, por manejos forestales de raleo selectivo y cortes en hoyos de luz

\section{CUADRO 7}

Microartrópodos del suelo en tratamiento "hoyo 2". Frecuencia de presentación en muestras (\%), densidad media en $\mathrm{N}^{\mathrm{o}}$ individuos $/ 1.000 \mathrm{ml}$, desviación estándar y número total de individuos colectados. Soil microarthropods in the round spot $\mathrm{N}^{\circ} 2$ treated forest plot. Sample frequencies (\%), mean densities $\left(\mathrm{N}^{\mathrm{r}}\right.$ individuals $\left./ 1,000 \mathrm{ml}\right)$, standard deviations and total number of collected individuals.

\begin{tabular}{|c|c|c|c|c|}
\hline $\begin{array}{l}\text { Lanco-H } \\
\text { Ноуо } 2\end{array}$ & $\begin{array}{c}\text { Frecuencia } \\
\%\end{array}$ & $\begin{array}{c}\mathrm{N}^{\circ} \\
\text { individuos/ } \\
1.000 \mathrm{ml} \\
\text { Medias }\end{array}$ & $\begin{array}{c}\text { Desviación } \\
\text { estándar }\end{array}$ & $\begin{array}{c}\mathrm{N}^{\mathrm{o}} \\
\text { total } \\
\text { individuos } \\
\text { colectados }\end{array}$ \\
\hline Oribatida & 100 & 147,0 & 88,2 & 147 \\
\hline Prostigmata & 50 & 6,0 & 7,0 & 6 \\
\hline Tarsonemini & 10 & 1,0 & 3,2 & 1 \\
\hline Gamasina & 100 & 32,0 & 31,9 & 32 \\
\hline Uropodina & 40 & 7,0 & 10,6 & 7 \\
\hline Total Acarina & 100 & 193,0 & 105,3 & 193 \\
\hline Araneida & 10 & 1,0 & 3,2 & 1 \\
\hline Pseudoscorpionida & 20 & 2,0 & 4,2 & 2 \\
\hline Diplópoda & 30 & 3,0 & 4,8 & 3 \\
\hline Chilópoda & 10 & 1,0 & 3,2 & 1 \\
\hline Collembola Entomobryomorpha & 80 & 41,0 & 56,7 & 41 \\
\hline Collembola Podurmorpha & 80 & 40,0 & 54,8 & 40 \\
\hline Collembola Symphypleona & 10 & 1,0 & 3,2 & 1 \\
\hline Hemíptera & 50 & 9,0 & 11,0 & 9 \\
\hline Thysanóptera & - & - & - & - \\
\hline Coleóptera Staphylinidae & 20 & 2,0 & 4,2 & 2 \\
\hline Coleóptera Leiodidae & 30 & 3,0 & 4,8 & 3 \\
\hline Coleóptera Ptiliidae & - & - & - & - \\
\hline Coleóptera Curculionidae & 10 & 1,0 & 3,2 & 1 \\
\hline Coleóptera Pselaphidae & - & - & - & - \\
\hline Coleóptera Tenebrionidae & - & - & - & - \\
\hline Coleóptera Dermestidae & - & - & - & - \\
\hline Coleóptera Carabidae & - & - & - & - \\
\hline Coleóptera Lathridiidae & - & - & - & - \\
\hline Larvas Díptera & 80 & 21,0 & 22,8 & 21 \\
\hline Larvas Coleóptera & 50 & 7,0 & 8,2 & 7 \\
\hline Larvas Lepidóptera & - & - & - & - \\
\hline Isópoda & - & - & - & - \\
\hline Copépoda & - & - & - & - \\
\hline Díptera (Imago) & 10 & 1,0 & 3,2 & 1 \\
\hline Formicidae & 50 & 5,0 & 5,3 & 5 \\
\hline Diplura Campodeidae & & & & \\
\hline Total Fauna & 100 & 331,0 & 195,9 & 331 \\
\hline
\end{tabular}


BOSQUE 25(1): 103-116, 2004 Variación en los microartrópodos del suelo, por manejos forestales de raleo selectivo y cortes en hoyos de luz

En resumen, respecto a los grupos de baja frecuencia, en bosque testigo un conjunto importante de taxa tiene mayores abundancias que en los tratamientos y, en todo caso, en testigo se encuentra el mayor número de taxa, varios de los cuales faltan en uno u otro tratamiento o en ambos, por lo que para estos grupos se puede hablar de empobrecimiento cuantitativo y cualitativo en los tratamientos.

Análisis de taxa con frecuencias y abundancias elevadas: se realizaron comparaciones, mediante análisis de varianza, entre grupos de muestras testigo con cada caso de tratamiento. En el caso de hoyos de luz, como se tomaron dos subgrupos en hoyos distintos, se probó primero si estos diferían o no entre sí.

En el cuadro 8 y 9 se dan los resultados de los contrastes probados.

Se puede observar que de los cinco casos probados, sólo larvas de Díptera no presentan diferencias significativas entre hoyo-1 y hoyo-2. En los otros 4 casos, significativos, se trata de medias mayores en taxa de hoyo- 1 respecto a hoyo-2. Con estos resultados inferimos que, en cuanto a estos taxa más representativos, ambos hoyos no son iguales y, por lo tanto, en las comparaciones posteriores deberán considerarse por separado.

En el cuadro 9 se da el resultado de los análisis de varianza entre testigo y hoyo-1 u hoyo-2.

Se observa en el contraste testigo/hoyo-1 que Collembola Poduromorpha y Entomobryomorpha son significativamente diferentes. Con los datos cuantitativos correspondientes (cuadros 3 y 6) se encuentra que Entomobryomorpha es más abundante en grupo testigo y Poduromorpha es más abundante en tratamiento hoyo. Los cuatro taxa restantes no difieren significativamente.

En la comparación testigo/hoyo-2, por el contrario, los únicos grupos que no difieren significativamente son los dos grupos de colémbolos, todos los demás taxa difieren muy significativamente, observándose para todos ellos un empobrecimiento de la abundancia en hoyo-2 (cuadros 3 y 7).

El último contraste se planteó entre grupo testigo y el grupo de tratamiento raleo, en el contraste con los datos de los grupos de mayor frecuencia detallados en el cuadro 10 .

Se puede observar que, con la excepción de Poduromorpha, los otros cinco taxa son diferentes

\section{CUADRO 8}

Resultados de análisis de varianza para abundancias de cinco taxa de frecuencias elevadas entre los subgrupos de hoyos.

Analysis of variance for the abundance of five frequent taxa tested in two different sized plots (round spot clear-cutting $\mathrm{N}^{\mathrm{r}} 1$ and $\mathrm{N}^{\mathrm{r}} 2$ ).

\begin{tabular}{|l|c|c|c|c|}
\hline Taxa & $\mathrm{F}$ & $\mathrm{p}-1$ & $\mathrm{n}-\mathrm{p}$ & Significación \\
\hline Oribatida & 5,18 & 1 & 18 & $\mathrm{~S}$ \\
\hline Total Acarina & 6,38 & 1 & 18 & $\mathrm{~S}$ \\
\hline Poduromorpha & 18,07 & 1 & 18 & $\mathrm{SS}$ \\
\hline Larvas Díptera & 0,64 & 1 & 18 & $\mathrm{NS}$ \\
\hline Total Fauna & 6,54 & 1 & 18 & $\mathrm{~S}$ \\
\hline
\end{tabular}

$\mathrm{S}=\mathrm{p}<0,05 ; \mathrm{SS}=\mathrm{p}<0,01 ; \mathrm{P}>0,05$.

significativamente. En todos estos casos las medias son menores en el tratamiento raleo (cuadros 3 y 4), comprobándose entonces un empobrecimiento de las abundancias de estos taxa dominantes y más representativos, que se puede atribuir al tratamiento.

Mediciones de los cambios en biodiversidad: en el cuadro 11 se dan para cada conjunto de muestras los valores de:

A) Número de taxa presentes;

B) $\mathrm{Su}$ índice de diversidad compuesta ( $\mathrm{H}^{\prime} \mathrm{de}$ Shannon);

C) Su índice de equiparidad (evenness), mediante el índice J de Pielou.

Se puede observar que el número de taxa es máximo en bosque testigo, disminuyendo en todos los tipos de tratamiento. En cuanto a la diversidad (H') se observa que es ligeramente mayor en raleo y hoyo-2, a pesar de la baja en el número de taxa, lo que se atribuye entonces al aumento de la equiparidad, como se corrobora en los índices J respectivos. Esta situación parece ser el resultado de una disminución relativa de la abundancia de los taxa de mayores densidades, dando por resultado una distribución más simétrica y, por ende, 
BOSQUE 25(1): 103-116, 2004

Variación en los microartrópodos del suelo, por manejos forestales de raleo selectivo y cortes en hoyos de luz

\section{CUADRO 9}

Resultado de análisis de varianza para abundancias de seis taxa con frecuencias muestrales elevadas, en contrastes entre bosque testigo con hoyo-1 y hoyo- 2 .

Analysis of variance for the abundance of six frequent taxa tested in the control forest plot with two treated plots (round spot $\mathrm{N}^{\mathrm{r}} 1$ and $\mathrm{N}^{\mathrm{r}} 2$ ) of different sizes.

\begin{tabular}{|l|c|c|c|c|}
\hline Testigo/Hoyo-1 & F & p-1 & n-p & Significación \\
\hline Oribatida & 1,48 & 1 & 28 & NS \\
Gamasina & 0,44 & 1 & 28 & NS \\
Total Acarina & 2,46 & 1 & 28 & NS \\
Entomobryomorpha & 5,91 & 1 & 28 & S \\
Poduromorpha & 14,67 & 1 & 28 & SSS \\
\hline Total Fauna & 0,36 & 1 & 28 & NS \\
\hline
\end{tabular}

\begin{tabular}{|l|r|c|c|c|}
\hline Testigo/Hoyo-2 & F & p-1 & n-p & Significación \\
\hline Oribatida & 15,54 & 1 & 28 & SSS \\
Gamasina & 7,85 & 1 & 28 & SS \\
Total Acarina & 21,71 & 1 & 28 & SSS \\
Entomobryomorpha & 2,86 & 1 & 28 & NS \\
Poduromorpha & 0,14 & 1 & 28 & NS \\
\hline Total Fauna & 15,37 & 1 & 28 & SSS \\
\hline
\end{tabular}

$\mathrm{S}=\mathrm{p}<0,05 ; \mathrm{SS}=<0,01 ; \mathrm{SSS}=\mathrm{p}<0,001 ; \mathrm{P}>0,05$.

\section{CUADRO 10}

Resultados de análisis de varianza entre grupos testigo y tratamiento de raleo selectivo para 6 taxa de mayor frecuencia.

Analysis of variance for the abundance of six frequent taxa tested in the control and treated plot (selective thinning).

\begin{tabular}{|l|c|c|c|c|}
\cline { 2 - 5 } \multicolumn{1}{c|}{} & F & p-1 & n-p & Significación \\
\hline Oribatida & 16,50 & 1 & 38 & SSS \\
\hline Gamasina & 4,78 & 1 & 38 & S \\
\hline Total Acarina & 15,83 & 1 & 38 & SSS \\
\hline Entomobryomorpha & 4,31 & 1 & 38 & S \\
\hline Poduromorpha & 3,71 & 1 & 38 & NS \\
\hline Total Fauna & 14,30 & 1 & 38 & SSS \\
\hline
\end{tabular}

$\mathrm{S}=\mathrm{p}<0,05 ; \mathrm{SSS}=\mathrm{p}<0,001 ; \mathrm{P}>0,05$.
CUADRO 11

Estimaciones de número total de taxa $(\mathrm{N})$, de diversidad compuesta (H') y de equiparidad (J) para bosque testigo y tratamientos forestales tipo raleo y tipo hoyo.

Higher taxa richness, Shannon-Weaver $\mathrm{H}^{\prime}$ index and J evenness index, for the native forest and treated plots.

\begin{tabular}{|l|c|c|c|}
\cline { 2 - 4 } \multicolumn{1}{c|}{} & $\mathrm{N}^{\mathrm{o}}$ taxa & $\mathrm{H}^{\prime}$ & $\mathrm{J}$ \\
\hline $\begin{array}{l}\text { Bosque testigo } \\
\text { Tratamiento } \\
\text { Raleo } \\
\text { Tratamiento }\end{array}$ & 28 & 2,59 & 0,54 \\
$\begin{array}{l}\text { Hoyo-1 } \\
\text { Tratamiento }\end{array}$ & 15 & 2,34 & 0,62 \\
$\begin{array}{l}\text { Hoyo-2 } \\
\text { Tratamiento } \\
\text { Hoyo-1 + Hoyo-2 }\end{array}$ & 22 & 2,78 & 0,64 \\
\hline
\end{tabular}


BOSQUE 25(1): 103-116, 2004

Variación en los microartrópodos del suelo, por manejos forestales de raleo selectivo y cortes en hoyos de luz

los citados aumentos de equiparidad y diversidad. Este caso es diferente del encontrado en el trabajo en bosque chilote siempreverde (23) en que bosque testigo tenía mayores valores de diversidad y equiparidad que todos los tratamientos. Lo que sí se repite en ambos tipos de bosque es la disminución del número de taxa en todos los tratamientos sin excepción.

Resumiendo los resultados, se encontró que para los 28 taxa de microartrópodos hallados en los suelos, se analizó por separado un subgrupo de baja frecuencia, de los cuales 8 taxa que se reproducen en bosque nativo no se presentan en los sectores tratamiento (los dos tipos de manejo), y además las abundancias de los que quedan suelen ser allí mucho menores. Por otra parte, el otro subgrupo de taxa de frecuencias elevadas, (5 ó 6 según los casos) en su gran mayoría muestran densidades medias significativamente menores en los terrenos sometidos a tratamiento, respecto del bosque nativo. Estos datos se apoyan en 60 muestras tomadas al azar, con tamaños habituales a este tipo de fauna, de modo que representan bien la varianza del sistema. Hacemos notar que este trabajo se refiere sólo a un estado del sistema en la escala temporal, y que son esperables cambios estacionales o a largo plazo, los que sería deseable estudiar a futuro; sin embargo, para el tipo de biocenosis estudiado, y con el muestreo y planteamientos empleados, los datos son ciertamente un buen índice sobre los cambios que provocan los manejos citados, en relación a la situación no perturbada de bosque nativo.

\section{CONCLUSIONES}

- El contenido de agua en las muestras, en porcentaje respecto al peso seco, es elevado, variando entre $130 \%$ y $135 \%$. Los contenidos de agua (g) de las muestras son significativamente mayores en los grupos muestrales del tratamiento "hoyos de luz", respecto al bosque testigo; en cambio, las diferencias no son significativas entre bosque testigo y tratamiento "raleo selectivo" (cuadros 1 y 2 ).

- En bosque testigo se encontraron 28 taxa de microartrópodos, los que en los tratamientos bajan a cifras entre 17 y 22 .
- Para el grupo de microartrópodos de frecuencias bajas, se encontró en los dos tipos de tratamiento una disminución importante en el número absoluto de taxa (faltan 8 taxa en uno $\mathrm{u}$ otro tratamiento respecto al bosque testigo); además, en el resto de los taxa, comunes a tratamiento y testigo, se encuentra una baja importante de las abundancias en ambos tratamientos. Se puede hablar entonces de empobrecimiento cuantitativo y cualitativo de este grupo de microartrópodos en ambos tipos de tratamientos de manejo silvícola.

- Para los taxa dominantes (frecuencias muestrales $>80 \%$ y densidades elevadas), de presencia común en testigo y tratamientos (5 ó 6 según los casos), se encontró en comparaciones de abundancias entre testigo y tratamientos, lo siguiente:

- contraste bosque testigo/raleo: 5 de los 6 taxa dominantes son diferentes significativamente y en todos esos casos las densidades medias son menores en el tratamiento que en bosque testigo. La excepción fue Collembola Poduromorpha (NS) (cuadro 10).

- contraste tratamiento/ hoyo-1: cuatro de los seis taxa difieren significativamente del testigo, presentando todas la densidades más bajas en el tratamiento. (cuadro 9).

- contraste testigo/hoyo-2: sólo dos de los seis taxa probados difieren significativamente, siendo uno de ellos más abundante en testigo y el otro más abundante en hoyo-2 (cuadro 9).

Se trataron separadamente los subgrupos de hoyo-1 y hoyo-2, ya que presentaron diferencias significativas en las abundancias de 4 de los 5 taxa dominantes (cuadro 8).

O sea, para la mayoría de los taxa dominantes se encuentra una baja significativa de las abundancias en los tratamientos; hace excepción el hoyo-2, que es de intervención más reciente y aun más parecido al bosque de origen.

- En cuanto al índice de diversidad H', es algo mayor en raleo y hoyo-2 que en testigo, efecto provocado por la citada disminución en los tratamientos de las abundancias de los grupos dominantes, lo que aumenta la simetría de la distribución, como se ve en los mayores valores del índice $\mathrm{J}$ de equiparidad. 
BOSQUE 25(1): 103-116, 2004

Variación en los microartrópodos del suelo, por manejos forestales de raleo selectivo y cortes en hoyos de luz

\section{BIBLIOGRAFIA}

(1) ARMESTO, J. Mitos y realidades del bosque nativo chileno. Rev. Chilena Hist. Nat. 1992. Vol. 65, p. 173-176.

(2) OTERO, L. A., A. CONTRERAS, A. BARRALES. Efectos ambientales de diferentes tipos de cortas en bosque nativo. El caso de las cortas de protección en fajas. Ciencia e Investigación Forestal. 1994. Vol. 8, Nº p. $87-118$

(3) OTERO, L. A., A. BARRALES, A. CONTERAS. Crecimiento inicial de plantaciones de raulí (Nothofagus alpina) en los métodos de protección en faja y protección uniforme, en el área de Neltume, Coñaripe. Ciencia e Investigación Forestal. 1995, Vol. 9, N² 2, p. 177-189.

(4) OTERO, L. A., L. CONTRERAS, L. BARRALES. Efectos sobre el suelo en el sistema de cortas en fajas y protección uniforme. Superficie de suelo alterada, densidad del suelo y escurrimiento superficial de agua, en el tipo forestal Coihue-Raulí-tepa. INFOR. Valdivia. 1996. Informe interno, p. 1-16 y 131-138

(5) HAWLEY, R., D. SMITH. Silvicultura práctica, $2^{\mathrm{a}} \mathrm{Ed}$ Barcelona: Omega S.A. 1982, p. 405.

(6) PROBST, J., T. CROW. Integrating biological diversity and resource management. Journal of Forestry. 1991. Vol. $89, \mathrm{~N}^{\mathrm{o}} 2$, p. $85-102$

(7) OCAÑA-VIDAL, J. Ordenación de bosques naturales mediante franjas protectoras. UNASYLVA 1992. Vol. 43 , p. 27-35.

(8) KAUFMANN, M. Soil temperature and drought effect on growth of Monterrey pine. Forest. Sc. 1977. Vol. 23, p. 317-324.

(9) COATES, K., J. EMMINGHAM, A. RADOSEVICH Conifer seedling success and microclimate at different levels of herbs and schrubcover in British Columbia. Can. J. of Forestry. 1991. Vol. 21, p. 858-866.

(10) DONOSO, C. Ecología Forestal. El bosque y su medio ambiente. Ed. Universitaria. Santiago. Chile. 1990, p. 369.

(11) GROSSE, H. Crecimiento de una plantación de Raulí y Roble bajo dosel, en dependencia del grado de luminosidad y fertilización. Ciencia e Investigación Forestal. INFOR. Chile. 1988. Vol. 2, No 5, p. 13-30.

(12) RONCO, F. Influence of light intensity on survival of planted Engelman spruce. Forest. Sc. 1970. Vol. 16 p. 331-339.
(13) GAYOSO, J. Pérdida de la productividad del sitio por efecto del madereo. Actas reunión "Evaluación de la productividad de sitios forestales". Univ. Austral. Chile. 1982, p. 7.

(14) GAYOSO, J., A. IROUMÉ. Daño en suelos forestales asociado a faenas de madereo. Medio ambiente. 1989. Vol. 10, $\mathrm{N}^{\mathrm{o}}$ 1, p. 70-79.

(15) WERT, S., B. THOMAS. Effects of skids roads on diameter, height and volume growth in Douglas fir. Soil Cs. Am. J. 1981. Vol. 45, p. 629-632.

(16) KRAG, R., K. HIGGINBOTHAM K., R. ROTHWELL. 1986. Logging and soil disturbance in southeast British Columbia. Can. J. For. Res. 1986. Vol. 16, p. 1345-1353.

(17) MALMER, A., H. GRIP. Soil disturbance and loss of infiltrability caused by mechanised and manual extraction of tropical rainforest in Sabah Malaysia. Forum Ecol. And Management. 1990. Vol. 38, p.15-23.

(18) INFOR. Mapa preliminar de Tipos Forestales. CORFO 1967. Santiago. Chile, p. 35.

(19) DONOSO, P., C. DONOSO, V. SANDOVAL. Proposición de zonas de crecimiento de renovales de roble (Nothofagus obliqua) y raulí ( $N$. alpina) en su rango de distribución natural. BOSQUE. 1993. Vol. 14, No 2, p. 85-93.

(20) WALLWORK, J. The distribution and Diversity of Soil fauna. London/New York/San Francisco: Academic Press. 1976 , p. 355

(21) HUHTA, V., E. KARPINNEN, M. NURMINEN, A. VALPAS. Effect of silvicultural practices upon arthropod annelid and nematode populations in coniferous soils. Ann Zool. Fenn. 1967. Vol. 2, p. 87-143.

(22) COVARRUBIAS, R. Comparación de fauna de microartrópodos entre bosque nativo y plantaciones de Pinus radiata de reemplazo, en biótopos equivalentes. Acta Entomológica Chilena, 1993, Vol. 18, p. 41-51.

(23) COVARRUBIAS, R., A. CONTRERAS. Efecto de manejos forestales del bosque siempreverde chilote, sobre los microartrópodos del suelo. BOSQUE. 1999. Vol. 20, $\mathrm{N}^{\mathrm{o}} 2$, p. 25-38.

(24) DAGNELIE, P. Théorie et Méthodes statistiques. Edit. Duculot. Gembloux. Belgique. 1970, p. 1-451.

(25) PIELOU. An introduction to mathematical Ecology. Wiley Interscience. N. York. 1969, p. 1-355

(26) COVARRUBIAS, R. Notes on the dynamics and decomposition of leaves in a Nothofagus pumilio forest. Environmental monitoring and assessment. 1994. Vol. 29, p. 253-266. 